\title{
MARIONETTE: FROM TRADITIONAL MANIPULATION TO ROBOTIC MANIPULATION
}

\author{
I-Ming Chen Raymond Tay Shusong Xing Song Huat Yeo \\ School of Mechanical and Production Engineering \\ Nanyang Technological University \\ Singapore 639798 \\ E-mail: michen@ntu.edu.sg
}

\begin{abstract}
Marionettes are string-operated puppets. It is an ancient and universal form of performing art which still evolves slowly today. From the engineering perspective, the marionette is a wire- (or string-) driven multi-limbed under-actuated mechanism under gravity influence that exhibits rich kinematic and dynamic behaviours. This article introduces the evolution and the engineering aspect of traditional marionette design and manipulation skills. Based on the mechanics of marionette, a novel robotic marionette system that manipulates the puppet through mechatronic means is developed. The marionette may create life-like movements according to programmed motion commands issued from the computer and a motor-driven puppeteer mechanism. A simple demonstration of the robotic marionette performance is shown as well.
\end{abstract}

KEYWORDS: Puppet. Marionette history. Robotic marionette. Puppeteer mechanism.

\section{INTRODUCTION}

Puppetry and Puppet Theater are popular art forms having a long and fascinating heritage in many cultures. The survival of this art form is due to man's fascination with the inanimate object animated in a dramatic manner [1], and human's curiosity to "reproduce" an exact artificial copy of him (or her). Recent advances in the humanoid robots speak for this quest of curiosity. Most types of puppets in use today fall into four broad categories: hand (or glove) puppets, rod puppets, marionettes, and shadow puppets [1]. The glove puppet is used like a glove on the operator's hand. The rod puppet is held and moved by rods, usually from below but sometimes from above. The marionette is a puppet on strings, suspended from a control mechanism held by the puppeteer. Shadow puppets are usually flat cut-out figures held against a translucent, illuminated screen. There is also a variety of combinations among the four categories: hand-rod, glove-rod, rod-hand, and rod-marionette puppets. Though people are mostly interested in the theatrical and artistic content of puppetry, basic puppet fabrication and manipulation techniques follow the physical laws and engineering principles. From the engineering point of view, the puppet, the puppet control device and the puppeteer form an interesting complex mechanical system that embodies life-like movement of artificially made entities. However, it is rare to see engineering study of the puppet- 
making and manipulation skills. Though there are quite a few literatures dedicated to the practice of puppetry, specific puppet making and manipulation skills are usually learned from the puppet masters through apprenticeship, and are usually not publicly available. This article first introduces the evolution of traditional marionette design and manipulation skills from the engineering perspective. Then, a novel robotic marionette system (ROMS - RObotic Marionette Systems) developed in Nanyang Technological University that manipulates the puppet through mechatronic means based on the mechanics of marionette instead of human operation, is described. The marionette may create life-like movements according to programmed motion commands issued from the computer and a motor-driven puppeteer mechanism. The marionette is chosen as the object of study for three reasons: 1) mechanically, it is equivalent to a cable-operated multiple rigid body system that exhibits rich kinematic and dynamic behaviours; 2) theatrically, the marionette performances are graceful, charming, and sometimes mystic because of the "invisible" string control; 3) the marionette is versatile and can be simple as well as sophisticate in both construction and control. Through this novel system we hope to compliment the trade of traditional puppetry in a mechatronic approach.

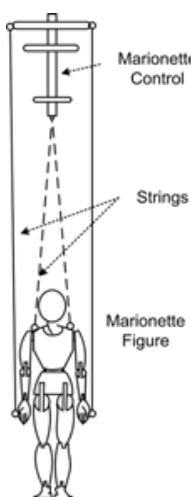

(a)
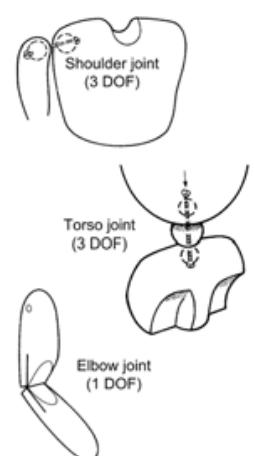

(b)

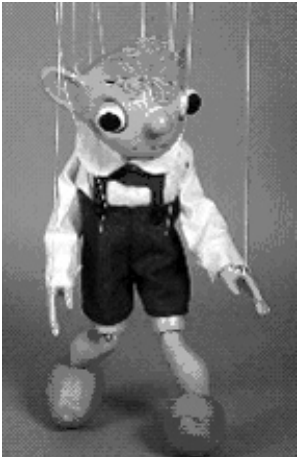

(c)

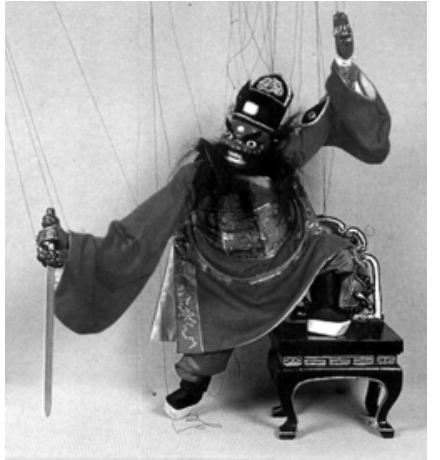

(d)

Fig.1: (a) Anatomy of a marionette. (b) Types of joints [1]. (c) Czech marionette (Hurvinek by Sotak). (d) Chinese marionette (Zhong Kui) [12]

\section{ANATOMY OF MARIONETTES}

The term "marionette" was first associated with string puppets in 16th century Europe. The origin of the word may be traceable to the Virgin Mary, often the principal character of puppet plays during the 1500s, either as a diminutive of "Maria," or in its literal translation "little Marys," from the French reference to the Virgin [2]. In China, it is called “Xuan Si Mu Ou” (懸絲木偶) or “Ti Xian Mu Ou” (提線木偶), which is a direct interpretation of the operation of the puppet. In the early history of Europe, the marionettes were used to entertain people - nobles or walk of all lives, whereas in China, the marionettes were mainly used for ceremonial purpose. The marionette theatres were used to entertain gods instead of ordinary people [3]. Hence, the appearance and fabrication techniques of marinettes have strong cultural influences. Regardless of cultural differences, the basic structure and anatomy of the marionettes are similar. In fact, this structure also changed very little through the history. A marionette usually 
consists of three essential elements: a puppet figure, a puppet control device, and a bundle of strings tied to the control device and various locations on the puppet figure as shown in Fig. 1(a). Together they form a string-operated multi-limbed mechanism.

\subsection{Puppet Figure}

The puppet figure is usually duplication or simplification of a human, an animal, or any kind of living creatures. The design of the figure follows three major steps: 1) artistic or theatrical feature development, 2) puppet movement or trick design and development, and 3) mechanical design of the puppet components. Mechanical design of the puppet covers the task of determining the number and shapes of limbs for the figure, types of joints connecting the limbs, and additional features on the puppet, such as blinking eyes, moveable mouthpieces, articulated palms for human figures, etc. The dexterity of puppet movement increases with more segments of limbs added to the figure, such as the inclusion of the torso segment in a human figure for the turning and bowing motions. The design of puppet joints is versatile. Joints with one degree of freedom (DOF) revolute motion, 2- and 3-DOF spherical motions are frequently used in the marionette. The DOFs in the joints are realized using different kind of connecting materials, such as leather, cord, or screw-eyes. Practical construction methods of puppet figures and joints can be found in [1, 4-11]. As shown in Fig. 1(b), the shoulder, torso, and limbs of a human puppet are all connected with each other through joints of different DOFs. A famous modern Czech marionette and a traditional Chinese marionette - Zhong Kui (鍾 魁), a ghost-fighting god [12], are shown in Fig. 1(c) and 1(d) respectively. The size of marionette figures performed in live is usually between 45 to $75 \mathrm{~cm}$ in height with consideration of the visual effect and convenience for the puppeteer operations.

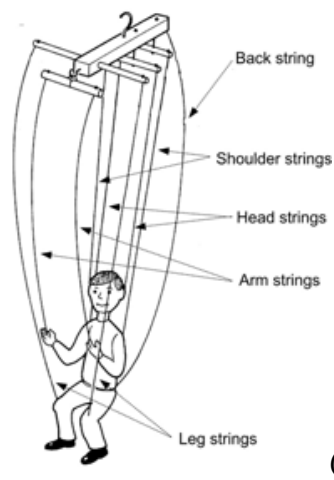

(a)

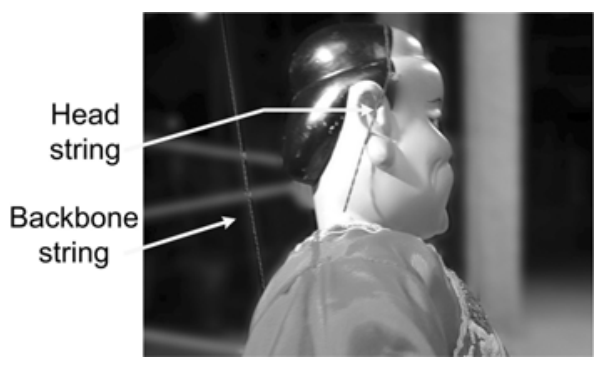

(b)

Fig. 2: (a) Functions of strings. (b) Head \& backbone strings on a traditional Chinese marionette.

\subsection{Strings}

In robotics terms, the strings for the marionette allow the puppeteer to "remotely operate" the puppet from a control device. By manipulating the control device and plucking the strings, the puppet will produce life-like motions. The strings act like "actuators" to the puppet. Hence, using proper type of strings for puppetry becomes an important issue. From our study [13], it has been noticed that western puppetry did not impose a standard type of strings used in the marionette, i.e., the control strings can be of any material. However, in the traditional Chinese puppetry, embroidery strings are 
frequently used. The embroidery strings are multi-threaded with little elasticity. Therefore, the strings will remain the same length under prolonged or large tension.

Functions of strings. The strings in a marionette can be classified into three categories: strings for support/reference, strings for motion control, and strings for special effect. The supporting strings hold most of the weight of the puppet figure and are usually kept stationary during the performance. In western human marionettes, the two shoulder strings usually serve this purpose (Fig. 2a). The traditional Chinese marionette includes a single reference string which acts as a backbone to the marionette (Fig. 2b). This is to ensure that the marionette is in an upright position. All remaining strings will take reference from this string. The strings attached to the arms, legs and the head of the figure are mainly for motion control purpose. The manipulation range of these strings attached to the limbs may be very large depending on the gesture of the figure. With the inclusion of head strings, the marionette may shake or nod his head. For a very basic human marionette, at least 8 strings are needed for full body motion: two for the shoulders (support), two for the arms, legs and the head each (control). Usually one back string is added to the western marionette having a torso joint (Fig. 2a). With this additional back string, the puppet figure may perform bowing motion.

Number of Strings. Using 8 to 9 strings in a generic marionette is only a rule of thumb. The actual number of strings used in a marionette depends on the desired performance of the figure. Western puppeteers tend to keep the number of strings to a minimum by using a counter-balanced design which allows gravity to hold a puppet in a "normal" pose when at rest. A typical western marionette with human figure uses 8 to 12 strings. Traditional Chinese marionettes normally use 16 to 24 strings, sometimes up to 50 strings, for a fully detailed human-like manipulation. These detail controls usually focus on the facial expressions, arm, and palm movements. In a Chinese marionette play called “Drunken Zhong Kui” (鍾魁醉酒) by the puppet master Mr. Huang Yique (黄奕缺) of Quanzhou (泉州), China, the figure of Zhong Kui (Fig. 1d) comprises 50 over strings. In the play, the marionette can perform very sophisticated movements like picking up wine cups, drinking the wine, pulling out and holding back the sword, etc.

\subsection{Control Device}

The control device for marionettes, or the "controller" here, is a simple mechanism held by the puppeteer to operate the puppet through a bundle of strings. The design and geometry of the marionette controllers vary in different cultures but evolve slowly with time. The Chinese use a simple platform type of control, termed “Gou Pai” (鈎牌 or hooked plate) which has passed down from Song dynasty for about a thousand years [14] whereas the Europeans generally adopt a cross platform control, sometimes called "airplane control" which took shape in the late $18^{\text {th }}$ century [15]. Such difference may probably due to the design and stringing of the marionettes and the philosophy of marionette performance. However, they do have a common feature of symmetric layout, which is also the fundamental feature of human body and most of the living beings.

2.3.1 Western marionette controllers

Unlike modern marionettes controlled by strings hung vertically using the balance of the tensions in the strings and the gravity, early in the history string-operated puppets 
were controlled by the strings laid horizontally. Figure 3(a) illustrates that puppets on the desktop controlled by horizontal strings were performed in the street in medieval France [16]. An early type of European marionette, known as "A La Planchette” in French or "fantoccini" in Italian was also controlled by horizontal strings as shown in an early $19^{\text {th }}$ century painting (Fig. 3b) [2]. It consisted of a string, secured to a post at one end, passing through the body of a jointed puppet or a group of puppets. Manipulation of the free end of string tied to the leg of a minstrel, caused movement and action thereby bringing the puppet to life.

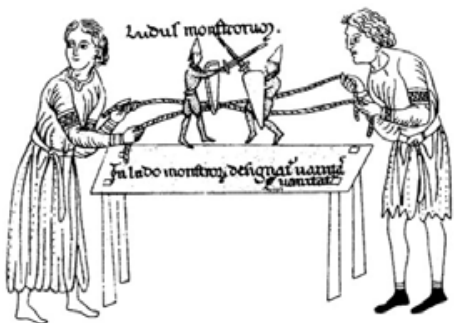

(a)

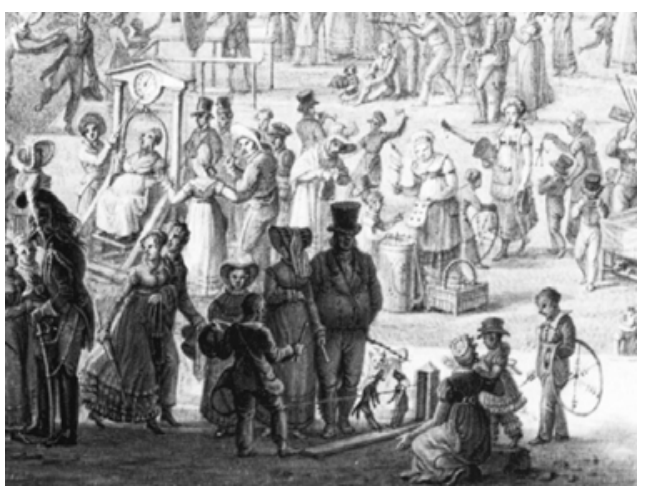

Fig. 3: (a) Puppets controlled by horizontal strings. (b) A La Planchette

Marionettes with vertical strings came in two forms in the $19^{\text {th }}$ century Europe: dramatic marionettes that were designed to perform in the dramatic repertoire and variety marionettes that were mainly for tricks and variety. Dramatic marionettes adopted a hybrid rod-string puppet design. In early days, the puppet figure was usually carved out of wood. Most puppets measured between 70 and $90 \mathrm{~cm}$ and, and when fully dressed and armed, their weight could be well over 10kg [15]. Using a rod attached to the head to support the weight is necessary. Control rods attached to the arms also allowed for strong clear expressive movement. However, as puppets adopted more dexterous design to give more vivid and lively performance for realism, the rod control became awkward for handling due to its rigid structure and number of rods used. In the late $19^{\text {th }}$ century, family-run puppet theaters in Europe gradually replaced control rods by strings on the puppets. The head rod was replaced with support strings after 1880 in English and German theaters. Nevertheless, some French and Italian theaters continued using the head rod until the companies ceased to function in 1930s [15]. Some rodstring marionette controllers used by European puppet theaters are illustrated in Fig.4.

Variety marionettes were those puppet figures that can dismember, give birth to other figures, expand or contract their bodies. Usually they were fully strung figures to maintain a sense of wonder because of their "invisible controls" [15]. From the late $18^{\text {th }}$ century, fully strung trick marionettes were usually controlled by one or more horizontal bars to which the strings were tied (Fig. 4). One bar took the hand and the head or support strings and the other, the ones for the legs. Later, British showmen mounted two or more of these horizontal bars onto a horizontal central bar making a single composite control which may have been one of the features that contributed to the success of the 
so-called "English marionettes". The vertical control for the all-string marionettes appeared in the end of $19^{\text {th }}$ century which was probably developed out of the rodmarionette grip. The control consisted of a vertical grip, with a horizontal bar at the bottom to take head strings, hand strings, and other special ones, and a rocking bar at the top for operation of the legs. The vertical control became standard in Germany and Britain, where it superseded the horizontal bars $[9,15]$. Some all-string marionette controllers used by European puppet theaters are illustrated in Fig. 4.

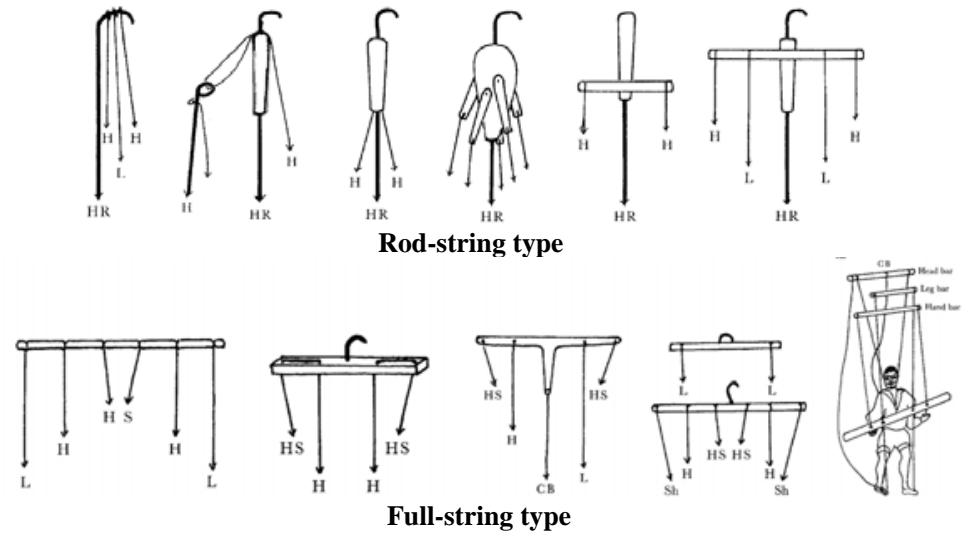

Fig.4: Various marionette controllers [adopted from 15]

The horizontal controller later proliferated in American in early $20^{\text {th }}$ century through European immigrants. Hence, we see a mixture of vertical and horizontal marionette controllers in modern puppet troupes. Figure 5(a) and 5(b) demonstrate typical vertical and horizontal controllers. They are also called airplane controls for the marionettes because their shapes resemble that of aircrafts. A variation of the airplane controller is the Angle controller invented by F. H. Bross [6,9]. (Fig. 5c) This controller has a central rod to be held by the puppeteer in tilted position. One major horizontal bar is pivoted to the central rod to produce rocking movement. Head strings are joined at additional horizontal bar on top and shoulder strings may join at the tail which is pivoted to the central rod as shown in the figure to produce swinging motion back and forth.

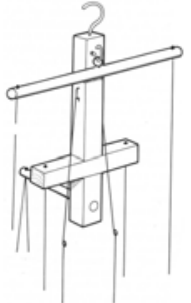

(a)

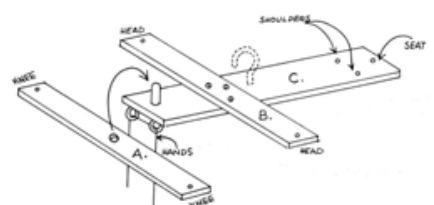

(b)

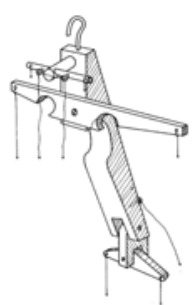

(c)

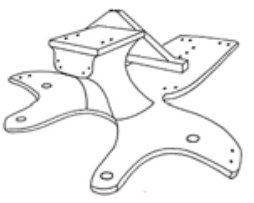

(d)

Fig. 5: (a): Vertical controller [1]. (b) Horizontal controller [5]. (c) Angle controller [6]. (d) Paddle controller [10].

A third type of marionette controllers is the paddle controller developed by W. A. Dwiggins $[17,18]$ as shown in Fig. 5(d). This tiny (a little over 6 inches) controller is 
shaped like a sweep-wing aircraft and can make the subtlest movements in 12" puppets. On the wing are strings for the head, forearm, and hands. On the main bar are strings for the forehead, shoulders, and back. The legs are controlled by a small piece of wood, vertical to the main bar and fixed on a pivot so the puppeteer can control the legs simply by rocking the bar with a finger [2]. For more variations of the vertical, horizontal, angle and paddle controllers, please refer to [9].

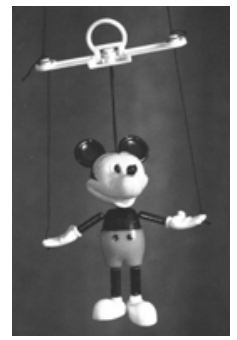

(a)

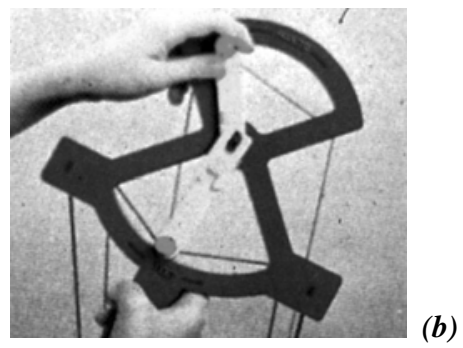

Fig.6: Toy type marionette controllers

The advancement of marionette controllers was fueled by commercialization. Until the late $19^{\text {th }}$ century, marionettes were not readily available to the public. It was not until the 1920s that the first commercially made marionette toys were available in the US. which were designed by Tony Sarg [19]. Because of the use of new materials, like plastics, for the puppets, the weight of the marionette can be further reduced and new types of marionette controllers can be developed. Simple bar control with a central ring allows children to use only one hand to handle the marionette [2] (Fig. 6a). An "action frame control” made of plastics was designed by Madison Ltd. in 1977 (Fig. 6b). The action frame control consists of two $90^{\circ}$ arc frame that the two control bars can slide through the slots in the respective arcs. The strings are attached to the central bars so that the puppeteer can manipulate the marionette by simply turning the central bars.

\subsubsection{Chinese marionette controller}

Chinese marionette theaters had been popular for more than a thousand years. Most of the repertoires of the marionette theater were adopted from those performed by the traditional Chinese theaters like Peking Opera. Hence, the marionette figures had strong connections to their human counterparts and the design of marionette figures was for realism. Therefore, the number of strings used by Chinese marionettes is usually more than the European marionettes. With so many strings to control, Chinese puppeteers made use of a controller known as the "Gou Pai", which is a single piece of plate on which all strings are nicely tied in systematical and symmetrical sequence (Fig. $7 \mathrm{~b}$ and 7c) [20]. The plate is made of wood or halved bamboo segment in rectangular shape about $25 \mathrm{~cm}$ long and $15 \mathrm{~cm}$ wide. The plate is attached to a long handle bar to be held by the puppeteer. A hook is nailed to the opposite face of the plate so that the controller can be hooked to a beam on the stage for the ease of manipulation. Because the number of strings is large and most of the puppet movements are produced by manually plucking the strings, using a single plate controller design is a practical and simple solution. The puppeteer's attention can be focus on the string manipulations rather than doing controller maneuver and string plucking at the same time. This marionette 
controller design has been passed down from Song Dynasty as illustrated by a Chinese painting in Song period (AD 960-1279) in Fig. 7(d) [21].
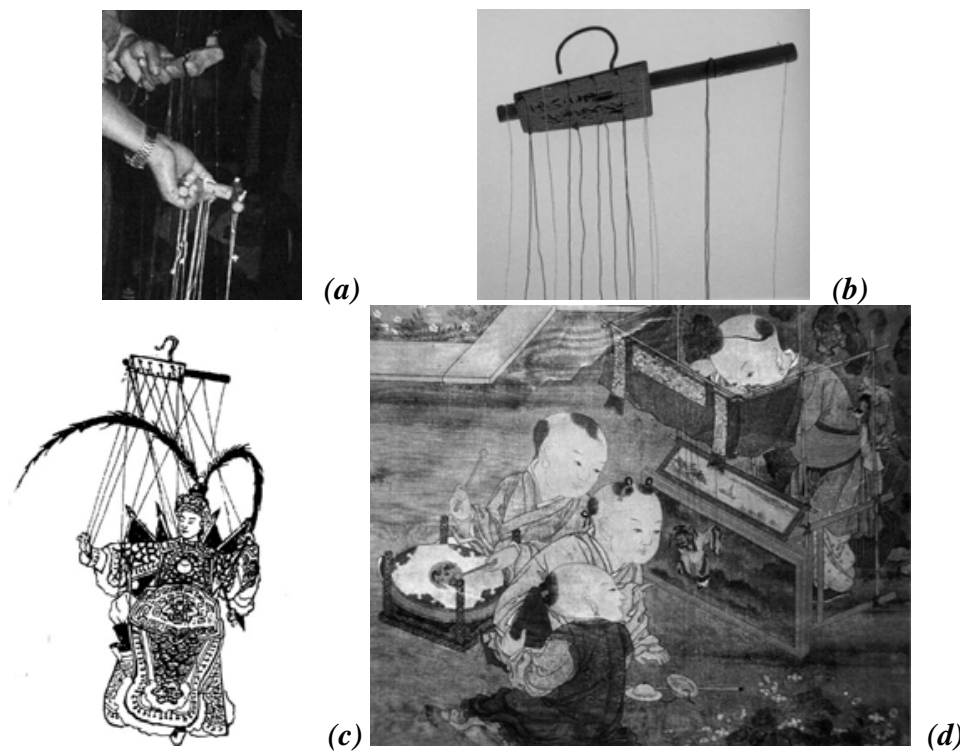

Fig. 7: (a) Burmese marionette controller. (b) Chinese Gou pai and (c) its operation [20]. (d) Puppet painting from Song Dynasty.

\subsubsection{Control devices from other cultures}

Figure 7(a) illustrates the controller of Burmese marionettes [22]. It is a palm-sized cross type control tightened with eight to ten strings. Because of its size, the puppeteer can manipulate the marionette with only one hand. For a sophisticate marionette with more than 10 strings, two such palm-sized controllers are employed. Instead of using any assistive device as marionette controllers, some puppeteers in India used their hands as the controller for the marionette [4]. Strings are tied to the fingers and the puppet figure. Moving the fingers and the hand will create puppet movements.

\section{CONVENTIONAL MARIONETTE MANIPULATION}

Unlike the humanoid robots where all limb joints are fully actuated and controlled, the marionettes are only "actuated" and controlled by a handful of strings attached to the limbs. Usually the number of control strings is less than the total degrees of freedom of the puppet figure. Assume that the puppet figure consists of $n$ rigid segments (or rigid bodies) and $j$ joints, and the DOF of each joint is $f_{j}$. According to Kutzbach criteria for computing the DOFs of spatial mechanisms [23], the DOF of the puppet figure without any strings connection, $M$, can be obtained by

$$
M=6(n-j)-\sum_{i=1}^{j} f_{i} .
$$

From the perspective of mechanisms, each string connection between the puppet figure and the controller can be considered as a "1-DOF" linear actuator that gives only the 
distance between the two connecting points. Therefore, it is necessary to have $M$ strings to fully control and manipulation of the puppet movements. However, in practice, the number of strings is always less than $M$, and there are uncontrollable DOFs in the marionette. If the number of strings is $k$, where $k<M$, the uncontrollable DOFs will be $M-k$. The marionette, then, can be described as an under-actuated string-operated multi-limbed mechanism. The configuration of these uncontrollable DOFs will be determined by the gravity or other external factors. As the marionette is under-actuated, manipulation and control of the marionette movement become a very tricky technique.

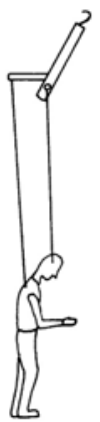

(a)

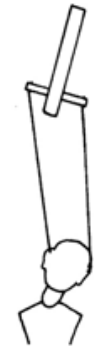

(b)

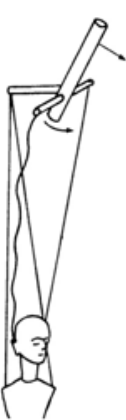

(c)

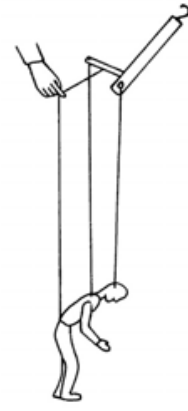

(d)

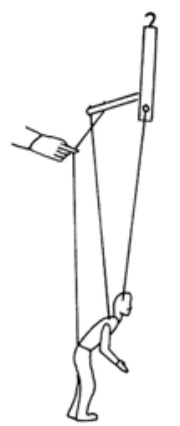

(e)

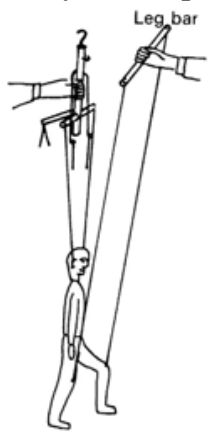

(f)

Fig. 8: Manipulating the controller to (a) nod the head; (b) incline the head; (c) turn the head; (d) bow the body; (e) keep the head upright; ( $f$ ) walk the puppet.
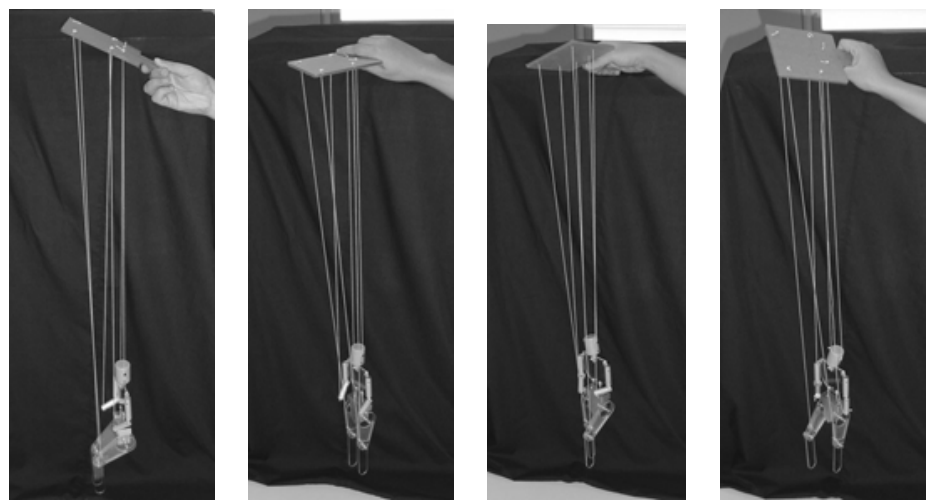

Fig. 9: Walking a 6-string puppet using self-made Gou Pai

Manipulation of the marionette through the controller usually is achieved in three manners: pure manoeuvring of the controller, plucking the strings by hands, and the combination of both. With a properly designed controller, a wide range of puppet movement can be achieved by simply tilting or turning the controller. For example, Figures 8(a), 8(b) and 8(c) show how to nod, incline, and turn the head respectively by maneuvering the controller [8]. Such kind of manoeuvring usually produces fine puppet movement because string lengths are unchanged. Figures 8(d), 8(e), 8(f) illustrate how to use the combined hand and controller motion to make the puppet bow [8], lift his head, and walk. To make large and prominent gestures, such as walking, plucking the strings or pulling the detachable horizontal bars is necessary (Fig. 8f). 
Usually one puppeteer can only operate one marionette from the controller unless the design of the controller can cater for multiple puppet figures. The puppeteer uses one hand to hold and maneuver the controller and the other to pluck or pull the strings and the detachable bars. With an ergonomically designed paddle controller (Fig. 5d), manoeuvring the controller and plucking the strings can be achieved using one hand simultaneously [10].

Manipulating the marionette using a Chinese Gou Pai is similar. Figure 9 shows a sequence of walking movement using a simple Gou Pai made in our laboratory with only six control strings. Because of the special layout, walking the puppet is achieved by rolling the Gou Pai clockwise and counter clockwise alternatively. Generally sophisticate movements can only be obtained by plucking various strings on the Gou Pai. Due to the large number of strings used in Chinese marionettes, maneuvering the Gou Pai becomes less obvious [20].

\section{ROBOTIC MARIONETTE SYSTEM}

The basic robotic marionette system consists of a puppet figure, a puppeteer mechanism hosting an array of inexpensive RC servo motors, pulleys and strings, a motor control network, and a Pentium II 200Hz PC running Linux operating system (Fig. 10) [13]. The puppet can produce life-like movements according to programmed motion commands issued from the computer through the puppeteer mechanism. Besides the standard human gestures and motions, the robotic marionette can "defy" the gravity and "fly" in the air to perform various stunts. Our focus on this system is on the development of the robotic puppeteer mechanism. We intend to make it portable and universal. Portability means that the system can be used as a standalone unit or can perform puppet shows side by side with puppeteers using manually operated marionettes. Universality means that the system adopts a generic and modular design so that different puppet figures can be mounted under the same puppeteer mechanism to perform different shows by simply changing the computer programs. Certain puppet figures with strong cultural, artisitc, or historical values can be mounted under the puppeteer mechanism to perform dynamic demonstration instead of static exhibition.

Three versions of robotic marionette systems, ROMS-I, II, and III [26] (Fig. 10) are developed. ROMS-I was the first prototype used to explore the pulley-motor concept for puppeteering tasks and the basic structure of the mechatronic system. The puppet figure of ROMS-I was modified from a small wooden human dummy and fishing wires were used as the control strings. ROMS-II and ROMS-III were improved versions of ROMSI with feedback from a professional puppet master and were developed simultaneously. The puppet figures of ROMS-II and III were life-like figures modified from toy soldiers with full clothes on for showing expressive behaviors. Standard embriodery threads were used as the control strings. The difference of the later two versions from ROMS-I is the design of the puppeteer mechanism. The pulleys were laid horizontally in ROMSI whereas they are in the vertical position in ROMS-II and III. The portability and modularity of the puppeteer mechanism were also taken into consideration. Basic specifications of the three ROMS are listed in Table 1. 


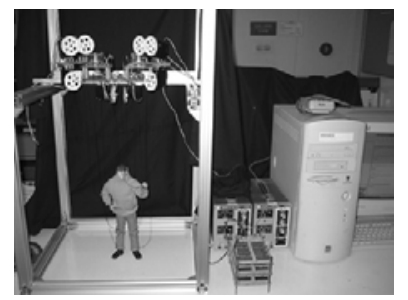

(a)

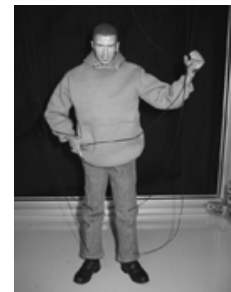

(b)

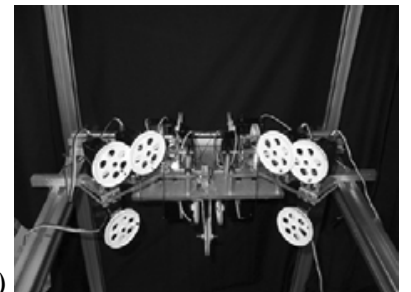

(c)
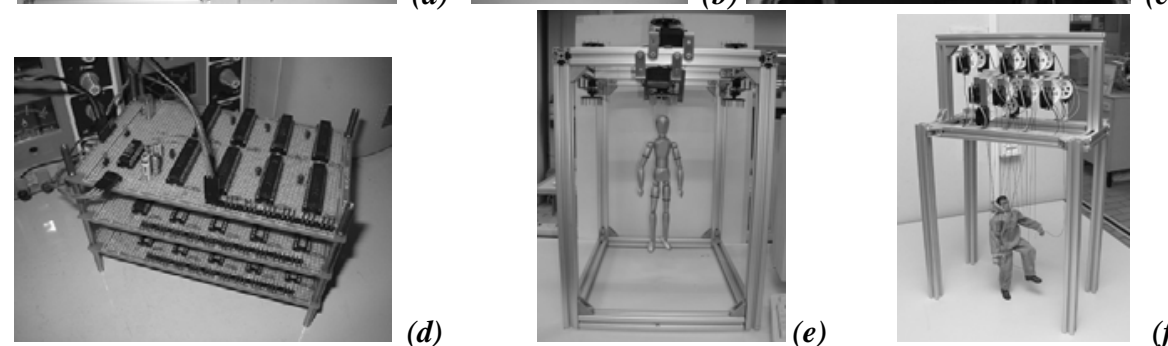

Fig. 10: (a) Overall marionette system (ROMS-II). (b) Puppet figure. (c) Robotic puppeteer Mechanism. (d) Motor control network. (e) ROMS-I (f) ROMS-III

\begin{tabular}{|c|c|c|c|}
\hline & ROMS-I [24] & ROMS-II [13,25] & ROMS-III [26] \\
\hline Motors & 8 & 16 & 16 \\
\hline Strings & 8 & $14^{*}$ & 16 \\
\hline DOF of puppet & 30 & 35 & 23 \\
\hline Links of puppet & 9 & 11 & 10 \\
\hline Joint of puppet & 8 & 10 & 9 \\
\hline Type of joint & 8 s-joint (3-dof) & 9 s-joint (3-dof) & 4 s-joint (3-dof) \\
& & 1 u-joint (2-dof) & 5 r-joint (1-dof) \\
\hline Height (cm) & 31.2 & 30.5 & 29.5 \\
\hline Weight (g) & 314 & 214 & 259 \\
\hline
\end{tabular}

Table 1: Specifications of ROMS-I, II, and III

\subsection{Puppeteer mechanism}

Here we focus on the puppeteer mechanism of ROMS-II and III, which becomes our reference design for further development. As we intend to develop the puppeteer mechanism as a generic platform for both Chinese and Western puppet performance, the maximum number of strings is set to be around 24 to 30 , and each string is to be individually controlled.

String-retracting device. The strokes of strings can be large due to the limb motion of the puppet. Various concepts had been proposed for the string-retracting device including the linear actuator, a linkage mechanism assisted pulley system, and a simple pulley-motor assembly [13]. Considering the stroke of the string, interference of the devices in motion, and the space required, the simple pulley-motor unit is chosen as the string-retracting device. Each string is controlled by one pulley-motor unit so that the length of the strings can be lengthened or shortened by simply rotating the pulley on the motor clockwise or counter clockwise. The pulley diameter is determined by the speed and resolution of puppet motion which require a balance between large motion range and fine smooth motion. The space constraint needs to be addressed as well. Based on the size of the puppet figure, the pulley diameter for ROMS-II and III is set to be $50 \mathrm{~mm}$. 
The selection of the motor has similar considerations. The motor chosen for the pulleymotor assembly is Parallax 900 continuous rotation servo motors. These motors accept speed control through continuous pulse-width-modulation (PWM) signals at $50 \mathrm{~Hz}$.

Mounting platform with extra DOF. A mounting platform combining the feature of Chinese and western marionette controllers is designed to house all pulley-motor units. As pulley-motor units can handle large as well as delicate string motions, motor units can remain stationary on the platform just like the all strings being tied to the Gou Pai. It is not necessary to mimic the manoeuvring of the controller as puppet masters do for this automated system. However, as strings drop vertically under gravity, performing puppet movements along the vertical plane, such as walking, nodding the head or bowing is much easier than sideway movements, such as arm opening and side-kicking, etc. Therefore, the two swinging armatures that can rotate horizontally are added to the mounting platform of ROMS-II to enable limbs to move sideways (Fig.10c). The movement of the two armatures are controlled by the servo motors. Three pulley-motor units controlling the arm and leg strings are mounted on each of the armature. The novel and extra degrees of freedom introduced on the mounting platform enable the marionette to produce more expressive behaviours. (Fig. 11a)

Motor/string functions. The assignment of limb movement to the corresponding pulley-motor unit is similar to the traditional marionette control. Among the total 16 motors installed on ROMS-II, there are two motors on each arm to control the elbow and hand respectively, three motors on each leg to control the knee, lower leg, and the ankle respectively, two for the head, one for the backbone string, one for the trick string for clapping hands, and two for the swinging armatures. The motor assignment on ROMS-III is similar to that of ROMS-II except that the two motors controlling the swinging armatures now control the shoulder of the puppet figure.
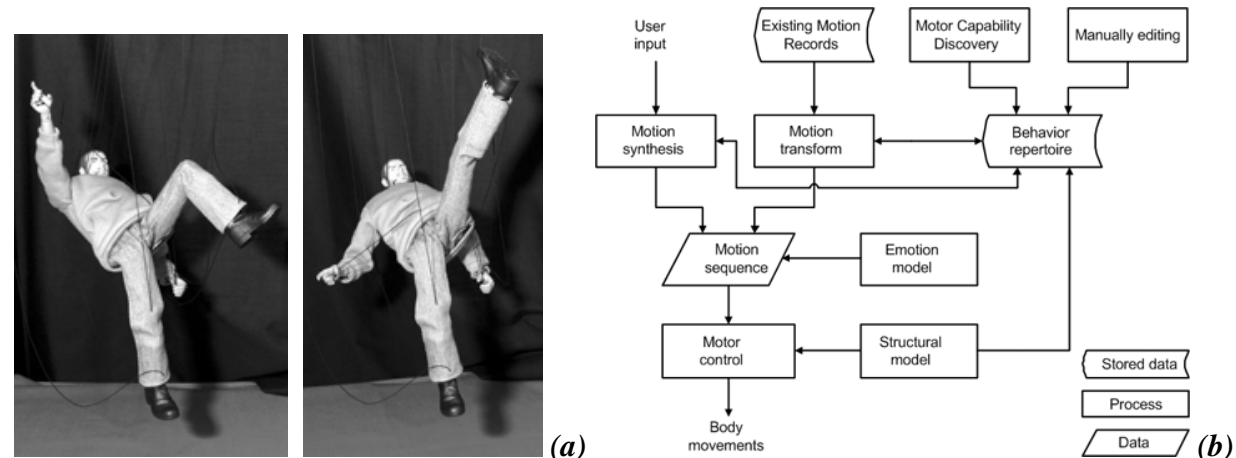

Fig. 11: (a) Puppet poses - Movie “Matrix"-like stunts. (b) Structure of Puppeteer software.

Motor layout issue. The design of the pulley-motor unit is modular. It is possible to reconfigure the pulley-motor arrangement for marionette with different number of strings and different characters. Due to the space and weight constraint, we adopt a layered motor mounting method to save the footprint of the puppeteer mechanism by stacking the pulley-motor units on top of each other. ROMS-II has two motor mounting layers on and below a single platform whereas ROMS-III has three motor mounting 
layers using extended fixtures.

4.2 Motor control network and interfacing

Motor control network. To cope with the large number (up to 30) of pulley-motor units, we develop a networked servo motor controller from scratch instead of off-theshelf products [24,25]. The networked controller allows the RC servo motor to be added or removed from the controller easily when the configuration of the marionette changes. The networked motor controller consists of 16 PICmicro 16F876 microcontrollers with each controlling one servo motor for position and velocity control. The microcontrollers communicate through a common I2C bus network. One idle microcontroller with no motor attached serves as the bridge between the PC and motor controller on the I2C bus through RS-232 serial port. The networked motor controller can incorporate heterogeneous devices compatible with the I2C bus protocol, such as various sensors.

Interface programming. Low level servo motor control based on PWM is coded and implemented on the PIC microcontroller using the proprietary assembly language for this class of chips. The firmware development tools include the MPLAB IDE integrated development environment software and the PICSTART Plus programmer from Microchip Corporation.

\subsection{Puppeteer software}

As the marionette is mechanically controlled by an array of pulley-motor units, coordination of pulley-motor actions to create marionette movements is achieved through a high level marionette motion generation program on the PC, called Puppeteer [25]. The Puppeteer software developed on the Linux PC for the robotic marionette provides an interface to accept motion control instructions from the user. The user has three ways to input and edit the robotic marionette movements: primitives, motion synthesis, and motion transformation. Different motion generation methods give the user some selections of varied workload in order to make the motion input process comfortable and effective [25]. The major modules of the puppeteer software are shown in Fig. 11(b). Except for the motor control module, most parts of the software were developed in C language and with GCC 2.2 under Redhat 6.1 Linux OS.

\subsection{Demonstration of robotic marionette behaviours}

The visual results of the robotic puppet engaging behavioural expression are captured in a short motion sequence. The postures shown in Fig. 12 are directly obtained by combining existing posture primitives. These postures illustrate the diversity of behaviours. Feedback from the collaborating puppet master is that pros and cons of ROMS ideally compliment traditional puppeteers. ROMS can control delicate marionette motion that a human may find very difficult to achieve manually. As human hands have limited capacity, the robotic marionette may produce some movement that cannot be achieved using the manual marionette controller. For example, the stunt of "motion freeze" in the mid air shown in the movie - Matrix was coded into ROMS-II by one of our students (Fig. 11b) to demonstrate that our marionette can "fly". However, ROMS does have drawbacks, mainly in its dynamic behaviour. When the string motion becomes very drastic, the marionette may swing in undesirable directions frequently in 
addition to its controlled movement [27]. This is fundamentally similar to the pendulum effect that needs to be compensated with more advanced control algorithms.

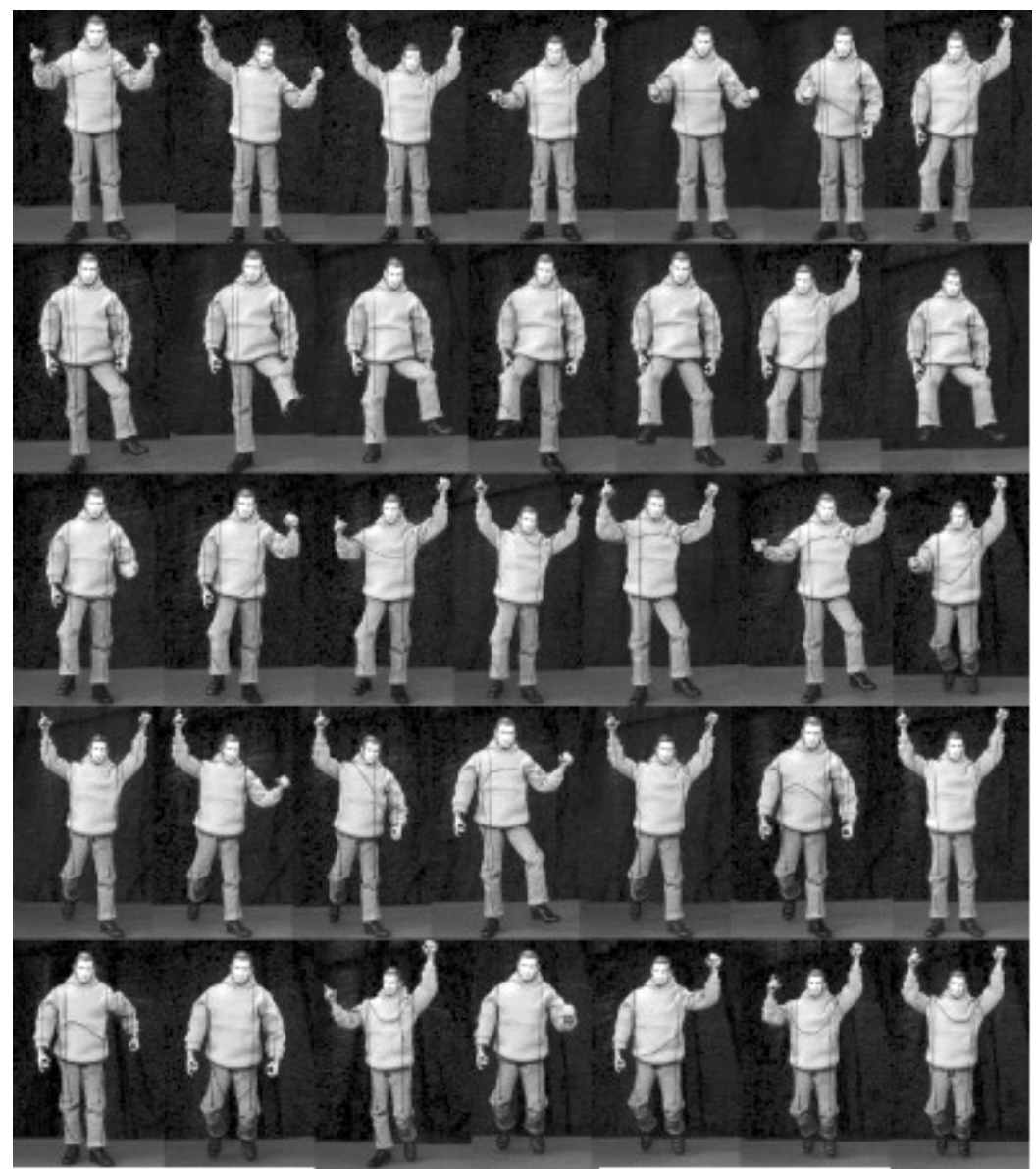

Fig. 12: ROMS-II Puppet postures

\section{V.CONCLUSION}

Marionette, a disappearing art form in Singapore and also in other countries, is a universal cultural theme. The puppeteer techniques involved become a cultural or national heritage; the making of marionette figures becomes fine folk arts. In the modern elementary education, puppetry is also a very effective tool for shaping children's personality, creativity, and thinking. This work examined the basic marionette techniques (design, anatomy, and manipulation) in engineering perspective. Through the development of robotic marionette systems, ROMS-I, II, and III, we would like to explore the possibility of infusing modern robotic and mechatronic technology into the traditional art form of marionette, evoke and stimulate public interests in this art form, and thus provide the puppetry and puppet theatre new element and new features. As addressed by the response from the puppeteer master, the range and features of 
robotic manipulation compliment the traditional marionette due to its system characteristics, eventually this kind of technology infusion may stay side by side with the traditional puppetry. From the mechanics point of view, multi-cable driven marionettes may provide us insight in developing cable-driven multiple rigid body systems for engineering applications as well.

Acknowledgement: The authors appreciate invaluable comments and suggestions on the puppeteer techniques provided by Ms. Beng Tian Tan of The Finger Players (www.fingerplayers.com), Singapore, and encouragement from Prof. M. Ceccarelli. Efforts made by other members in this project, S. K. Tan, Ronald Yeu, Stefan Künzler, Wei Ji, Wesley Chia, are also appreciated.

\section{REFERENCES}

[1] D. Currell. Puppets and Puppet Theatre, Crowood Press, Wiltshire, UK, 1999.

[2] D. E. Hodges. Marionettes and String Puppets Collector's Reference Guide, Antique Trader Books, VA, USA, 1998.

[3] K. D. Sun. Origin of Chinese Puppetry (傀儡戲考原). Shanghai Publishing Co., Shanghai, China, 1952. (Chinese)

[4] C. Flower, A. Fortney. Puppets: Methods and Materials, Davis Publications, MA, USA, 1983.

[5] G. Latshaw. The Complete Book of Puppetry, Dover Publications, NY, USA, 1978.

[6] O. Batek. Il Teatro Delle Marionette, Ottaviano, Milan, Italy, 1981.

[7] H. Fling. Marionettes: How to Make and Work Them, Dover Publications, NY, USA, 1973

[8] D. Currell. The Complete Book of Puppetry, Pitman Publishing, 1974.

[9] L. Coad. Marionette Sourcebook, Charlemagne Press, Vancouver, Canada, 1993.

[10] B. Frascone, D. Frascone. The Art and Technique of Marionette Making, Vol., France, 2002.

[11] M. Steven. Steven's Course in Puppetry. Charlemagne Press, Vancouver, Canada, 1997.

[12] Q. Liu, S. L. Jiang. Chinese Puppetry Art (中國木偶藝術). China World Language Publishing (中國世界語 文出版社), Beijing, China, 1993. (Chinese)

[13] B. K. Tay. Development of a Robotic Marionette System. Final Project Report, School of Mechanical and Production Engineering, Nanyang Technological University, 2003.

[14] Q. Y. Yang. History of Puppet Theater in Hong Kong. Cosmos Books, Ltd., Hong Kong, 2001. (Chinese)

[15] J. McCormick, B. Pratasik. Popular Puppet Theatre in Europe, 1800-1914, Cambridge University Press, UK,1998.

[16] M. Violette, History of French Puppetry, Proc. Int. Puppet Theatre Conf., Taipei, Taiwan, 1999, pp234-

261. (ISBN 957-02-4287-6)

[17] W. A. Dwiggins. Marionette in Motion, Puppetry Imprint, Detroit, USA, 1939.

[18] D. Abbe. The Dwiggins Marionettes, Abrams Inc., NY, USA, 1970.

[19] J. Bell. Strings, Hands, Shadows: A Modern Puppet History, Detroit Institute of Arts, USA, 2000.

[20] P. Tso. Puppet Theatre in Hong Kong and Their Origins, Hong Kong Municipal Government, 1987.

[21] Anon. Children playing with string puppets, Song Dynasty painting, National Palace Musuem, Taiwan.

[22] M. Thanegi. The Illusion of Life. White Orchid Press, Bangkok, Thailand, 1995.

[23] J. E. Shigley, J. J. Uickers. Theory of Machines and Mechanisms, 2nd ed., McGraw-Hill, NY, USA, 1995.

[24] S. S. Xing, I.-M. Chen. Design Expressive Behaviors for Robot Puppets, Proc. 7th Int'l Conf. Control, Automation, Robotics, Vision, Singapore, pp378-383, 2002.

[25] S. S. Xing. Behavior-Based Physical Agents as Information Display Devices, PhD Dissertation, School of Mechanical and Production Engineering, Nanyang Technological University, 2003.

[26] S. Künzler. Development of Programmable Puppeteer Mechanism for Robotic Marionette Theatre, Diploma Thesis, School of Mechanical and Production Engineering, Nanyang Technological University and Hochschule Rapperswil, Switzerland, 2003.

[27] K. Yamane, J K. Hodgins, H. B. Brown. Controlling a Marionette with Human Motion Capture Data. Proc. IEEE Int. Conf. Robotics Automation, Taipei, Taiwan, 2003, pp3834-3841. 